\title{
Liver injury in patients with COVID-19: clinical profiles, CT findings, the correlation of the severity with liver injury
}

\author{
Ping Lei $\mathrm{i}^{1,2} \cdot$ Lan Zhang $^{1,2} \cdot$ Ping Han ${ }^{1,2} \cdot$ Chuansheng Zheng ${ }^{1,2} \cdot$ Qiaoxia Tong $^{3} \cdot$ Haitao Shang ${ }^{4} \cdot$ Fan Yang ${ }^{1,2} \cdot \mathrm{Yu} \mathrm{Hu}^{5}$. \\ Xin $\mathrm{Li}^{1,2}$. Yuhu Song ${ }^{4}$
}

Received: 28 April 2020 / Accepted: 20 August 2020 / Published online: 4 September 2020

(c) Asian Pacific Association for the Study of the Liver 2020

\begin{abstract}
Background and aims Liver injury is found in some of patients with COVID-19. Liver injury of COVID-19 patients based on severity grading and abdominal radiological signs have not been reported until now. The aim of our study is to determine clinical profiles of the patients based on severity grading, describe abdominal radiological signs, and investigate the correlations of the severity with clinical profiles and radiological signs.

Methods This retrospective cohort study included 115 patients with COVID-19 from Jan 2020 to Feb 2020. Medical records of the patients were collected and CT images were reviewed.

Results Common clinical manifestations of patients with COVID-19 were fever (68.70\%), cough (56.52\%), fatigue (31.30\%); some of them had gastrointestinal symptoms (diarrhea, $12.17 \%$; nausea or vomiting 7.83\%; inappetence, $7.83 \%$ ). Abnormal liver function was observed in some of patients with COVID-19. Significant differences in the levels of AST, albumin,CRP were observed among different groups classified by the severity. Common findings of upper abdominal CT scan were liver hypodensity $(26.09 \%)$ and pericholecystic fat stranding $(21.27 \%)$; liver hypodensity was more frequently found in critical cases $(58.82 \%)$. The severity of COVID-19 correlated with semi-quantitative CT score of pulmonary lesions, CT-quantified liver/spleen attenuation ratio in patients with COVID-19.

Conclusions Some of the patients with COVID-19 displayed liver damage revealed by liver functional tests and upper abdominal CT imaging, and the severity of COVID-19 patients correlated with some of liver functional tests and CT signs; thus, it will allow an earlier identification of high-risk patients for early effective intervention.
\end{abstract}

Keywords COVID- $19 \cdot$ Liver $\cdot$ Clinical profiles $\cdot$ Tomography $\cdot$ X-ray computed

\section{Abbreviations}

CT Computed tomography

COVID-19 Coronavirus disease 2019

Ping Lei and Lan Zhang contributed equally to this study.

Electronic supplementary material The online version of this article (https://doi.org/10.1007/s12072-020-10087-1) contains supplementary material, which is available to authorized users.

Yuhu Song

yuhusong@163.com

Xin Li

1xwsry2014@163.com

1 Department of Radiology, Union Hospital, Tongji Medical College, Huazhong University of Science and Technology, Wuhan 430022, China

2 Hubei Province Key Laboratory of Molecular Imaging, Wuhan 430022, China
SARS-CoV-2 The severe acute respiratory syndrome coronavirus 2

2019-nCoV 2019 Novel coronavirus

ARDS Acute respiratory distress syndrome

MOF Multiple organ failure

DILI Drug-induced liver injury

ROI Regions of interest

ICC Intraclass correlation efficient

3 Department of Infectious Diseases, Union Hospital, Tongji Medical College, Huazhong University of Science and Technology, Wuhan 430022, China

4 Division of Gastroenterology, Union Hospital, Tongji Medical College, Huazhong University of Science and Technology, Wuhan 430022, China

5 Institute of Hematology, Union Hospital, Tongji Medical College, Huazhong University of Science and Technology, Wuhan 430022, Hubei, China 


$\begin{array}{ll}\text { RT-PCR } & \text { Reverse transcription-polymerase chain } \\ & \text { reaction } \\ \text { WBC } & \text { White blood cells } \\ \text { PLT } & \text { Platelet } \\ \text { LY } & \text { Lymphocytes } \\ \text { T-BIL } & \text { Total bilirubin } \\ \text { ALB } & \text { Albumin } \\ \text { PT } & \text { Prothrombin time } \\ \text { INR } & \text { International normalized ratio } \\ \text { Cr } & \text { Creatinine } \\ \text { CRP } & \text { C-reactive protein } \\ \text { IQR } & \text { Interquartile range } \\ \text { L/S } & \text { Liver-to-spleen } \\ \text { ALT } & \text { Alanine aminotransferase } \\ \text { AST } & \text { Aspartate aminotransferase } \\ \text { ALP } & \text { Alkaline phosphatase } \\ \gamma \text {-GT } & \gamma \text {-Glutamyl transpeptidase } \\ \text { ULN } & \text { Upper limit of normal } \\ \text { LLN } & \text { Lower limit of normal } \\ \text { UA } & \text { Uric acid } \\ \text { CHOL } & \text { Cholesterol } \\ \text { TG } & \text { Triglycerides } \\ \text { ACE2 } & \text { Angiotensin-converting enzyme 2 } \\ \text { NAFLD } & \text { Non-alcoholic fatty liver disease } \\ & \end{array}$

\section{Introduction}

2019 novel coronavirus disease (COVID-19) is a zoonotic illness caused by a new viral pathogen named as the severe acute respiratory syndrome coronavirus 2 (SARS-CoV-2), previously known as 2019 novel coronavirus(2019-nCoV) [1].The rapid spread of COVID-19 has created a global health crisis. Lung is considered as a major organ attacked by SARS-CoV-2. Besides the respiratory system, the infection of SARS-CoV-2 leads to various degrees of damage to other organs such as the liver, heart, kidney and gastrointestinal tract [2-5]. The majority of COVID-19 patients only suffered mild symptoms; while some COVID-19 patients progressed rapidly with acute respiratory distress syndrome (ARDS), and eventually resulted in multiple organ failure (MOF) $[1,6]$.

Previous studies showed that some of patients infected by SARS-CoV-2 had gastrointestinal symptoms, such as diarrhea, abdominal pain, inappetence, nausea and vomiting [7-9]. In addition, liver injury occurred in patients with COVID-19 [8-10].The abnormality of liver function tests was observed in some of COVID-19 patients at admission [11]. Pathological examination of COVID-19 patient showed hepatocyte degeneration accompanied by focal necrosis, moderate steatosis, infiltration of leukocytes into lobular and portal area, and sinusoidal congestion [12]. Thus, all these indicated liver involvement in patients infected by
SARS-CoV-2. While previous studies collected the data from the whole cohort of patients and some of them probably had chronic liver diseases.

Chest CT, a routine imaging tool for pneumonia diagnosis, plays a central role in the diagnosis and classification of COVID-19 pneumonia [13-15]. Moreover, radiological signs of chest CT varied with the course of diseases, and the change of radiological signs might be associated with the outcomes of diseases $[15,16]$. Liver injury in patients infected by SARS-CoV-2 might result in radiological change in liver. However, radiological findings of abdominal CT scan have not been explored so far. Thus, the aim of our study is to determine clinical profiles of the COVID-19 patients based on severity grading, describe abdominal radiological signs, and investigate the correlations of the severity with clinical profiles and radiological signs.

\section{Materials and methods}

\section{Study population}

Our retrospective study was performed at Cancer Center of Union Hospital and main campus of Union Hospital, Tongji Medical College, Huazhong University of Science and Technology from Jan 24, 2020 to Feb 17, 2020. 178 consecutive patients with COVID19 pneumonia were enrolled. The diagnosis of COVID19 patients are: (1) fever and/or respiratory symptoms; (2) positive nucleic acid of the virus (RTPCR analysis of throat swab specimens). A severity-grading system for patients with COVID19 was issued by National Health Commission of the People's Republic of China and National Administration of Traditional Chinese Medicine of the People's Republic of China. The severity of COVID19 patients is classified into four grades: mild, ordinary, severe and critical [13]. Mild cases: symptomatic infection includes fever, cough, sore throat, fatigue, headache or myalgia, but there were no pneumonia signs on chest imaging. Ordinary cases: symptomatic infection includes fever, cough, sore throat, fatigue, headache or myalgia, and pneumonia signs showed on chest imaging, meanwhile, these patients do not have any of the severe or critical symptoms and complications described below. Severe cases: the disease progresses to meet any of the following conditions: (1) significantly increased respiration rate: $\mathrm{RR} \geq 30 / \mathrm{min}$; (2) $\mathrm{SpO}_{2} \leq 93 \%$ from a finger oximeter; (3) $\mathrm{PaO}_{2} / \mathrm{FiO}_{2} \leq 300 \mathrm{mmHg}$; (4) patients with $>50 \%$ lesions progression within $24-48 \mathrm{~h}$ in pulmonary imaging. Critical cases: with any of the following conditions: (1) respiratory failure which requires mechanical ventilation; (2) shock; (3) accompanied by other organ failure that needs ICU monitoring and treatment. Inclusion criteria were as follows: (1) confirmed cases with COVID-19; (2) pneumonia manifestation on chest CT scan; (3) CT scan 
and laboratory examinations were performed within 2 days after admission. Exclusion criteria included: (1) patients who received antiviral treatment for more than 3 days before admission, such as interferon, chloroquine phosphate, arbidol, lopinavir/ritonavir, which may result in the alleviation of COVID-19 and side effects including GI symptoms and drug-induced liver injury, etc., $(n=30)$. (2) CT images of upper abdomen were not obtained $(n=19)$; (3) patients with hematological malignancy who were receiving chemotherapy $(n=6)$; (4) patients with severe underlying pulmonary diseases $(n=4)$; (5) patients with chronic liver diseases and liver cancer $(n=4)$ (Fig. 1).

\section{Data collection}

The pertinent data of the patients were collected from medical records and tabulated in a database. The pertinent data of the patients included demographic data; presenting symptoms and signs; medical history; laboratory tests; imaging findings; therapeutic process.

\section{Imaging technique and imaging analysis}

All CT examinations were performed with one of the following three scanners: 64-detector spiral CT scanner (Somatom Definition AS, Siemens, Germany), gemstone spectral CT scanner (Discovery CT750 HD, GE, USA), 16-detector spiral CT scanner (Activion 16, Toshiba, Japan). Images were reconstructed into lung window and soft tissue mediastinal window with a slice thickness of $1.5 \mathrm{~mm}, 2 \mathrm{~mm}$ or $5 \mathrm{~mm}$ and an interval of $1.5 \mathrm{~mm}, 2 \mathrm{~mm}$ or $5 \mathrm{~mm}$, respectively. The radiological evaluation included pulmonary involvement and abnormal signs of upper abdomen. All CT images were independently reviewed and the findings were recorded by two experienced radiologists who were unaware of clinical data. For semi-quantitative and qualitative imaging evaluation, discrepant evaluation was jointly re-evaluated to reach a final consensus [17-19]; whereas, intraclass correlation coefficient (ICC) was introduced to assess interobserver consistency for quantitative imaging evaluation. The severity of pneumonia was evaluated by a semi-quantitative scoring system, i.e., each of the 5 lung lobes was visually scored from 0 to 5 as: 0 , no involvement; $1,<5 \%$ involvement; 2, 5-25\% involvement; 3, 26-49\% involvement; 4, 50-75\% involvement; $5,>75 \%$ involvement. The total CT score was the sum of individual lobar scores and ranged from 0 (no involvement) to 25 (maximum involvement) [20, 21]. Radiologic signs reviewed in upper abdominal CT scan include: hepatomegaly, the density of liver, periportal edema, pericholecystic fat stranding, portal lymphadenopathy, biliary ductal dilatation, ascites. Liver hypodensity was defined when the density of liver is homogeneous or heterogeneous lower than or nearly equal to that of spleen. CT-quantified hepatic density was measured by liver-to-spleen (L/S) attenuation ratio. Hepatic and splenic $\mathrm{CT}$ attenuation values were measured using regions of interest (ROI) $>100 \mathrm{~mm}^{2}$ in area [22] on the PACS (CARESTRAM 3.1.S12.1, Canada). Two ROIs were in the right liver lobe, one ROI in the left liver lobe and three ROIs in the spleen. ROI should include a greater area of the liver and spleen whenever possible,

\section{The patients with COVID-19 who received CT scan and laboratory examination within 2 days}

after admission from January 24, 2020 to February 17, $2020(\mathrm{~N}=178)$

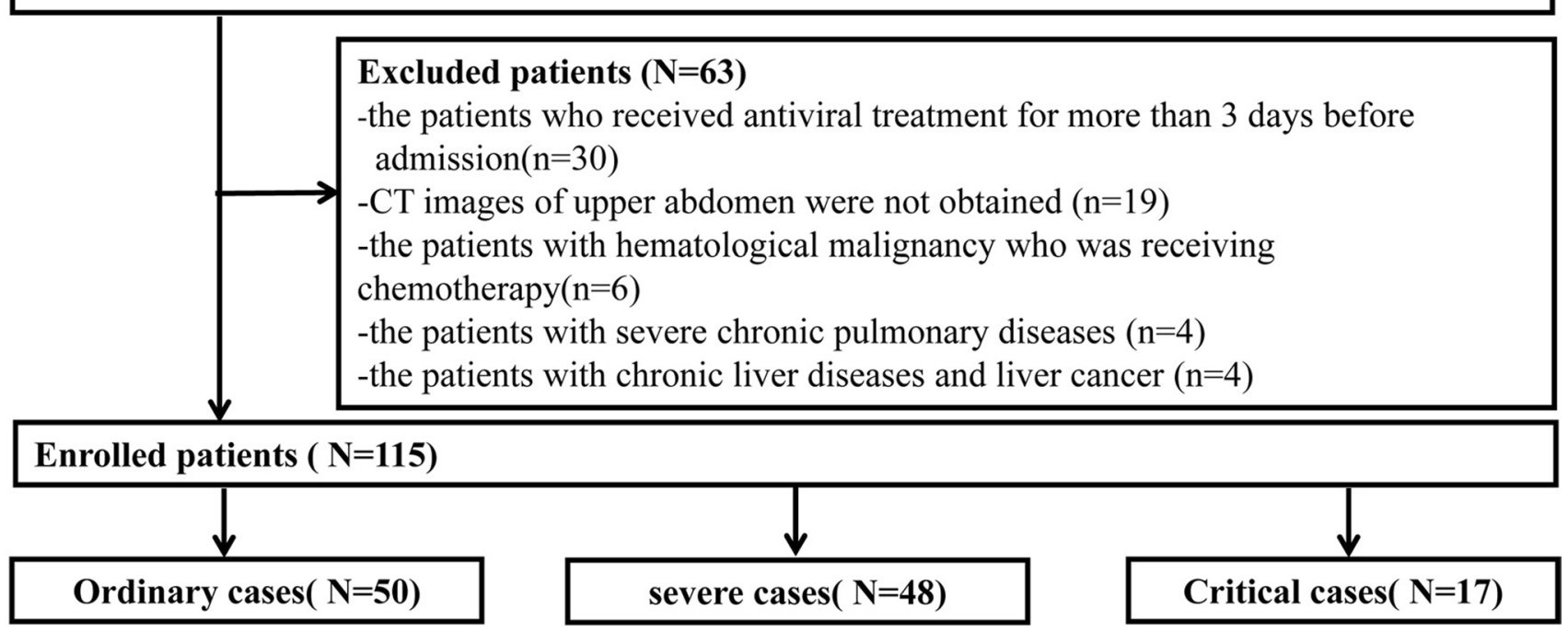

Fig. 1 Flowchart of the patients' selection, inclusion, and exclusion 
meanwhile ROI selection should exclude regions of hepatic vessels and bile ducts (Fig. S1). Then L/S ratio was calculated by taking mean $\mathrm{HU}$ measurement of liver lobe ROIs and dividing it by the mean splenic $\mathrm{HU}$ measurement of its ROIs (Fig.S1).

\section{Statistical analysis}

Continuous variables were expressed as mean and standard deviation for normal distribution data and median (25th-75th percentiles) if the distribution was skewed. Categorical variables were presented as count (percentage). The interobserver agreement for measurement of CT-quantified hepatic density was determined using the intraclass correlation efficient (ICC). The level of agreement was generally recognized as follows: poor, ICC $<0.20$; fair, $0.2<$ ICC $\leq 0.40$; moderate, $0.4<$ ICC $\leq 0.60$; good, $0.6<$ ICC $\leq 0.80$; and very good, $0.8<$ ICC $\leq 1.0$. The differences of categorical variables among groups were analyzed by Chi-square test (the Fisher exact test was used when the data were limited) and continuous variables by Kruskal-Wallis $\mathrm{H}$ test. The correlation of categorical variables was analyzed by Spearman correlation test and continuous variables by Pearson correlation test. All statistical tests were two-sided. $p$ value less than 0.05 was considered to indicate statistical significance. Statistical analyses were performed using SPSS version 22.0 (SPSS Inc., Chicago, Illinois, USA).

\section{Results}

\section{Clinical presentation}

115 patients with COVID-19 pneumonia were consecutively enrolled in our study. The patients were classified into mild group, ordinary group, severe group and critical group according to the severity [13]. Mild cases without pneumonia manifestation did not receive inpatient hospital care in Wuhan, China, so mild cases were not included in our study. Thus, the enrolled patients included ordinary cases (50 patients), severe cases (48 patients) and critical cases (17 patients) (Fig. 1). The median age of enrolled patients were 66 years (IQR: 60-70), there was 58 males and 57 females, with a male-to-female ratio of 1.02:1 (Supplementary Table 1). Significant difference in patients' age existed among different groups, which revealed that older patients were at higher risk for severe illness. Then, the clinical manifestations of the patients were analyzed, and the results showed fever in $68.70 \%$ (79/115) of the patients, cough in $56.52 \%$ (65/115), fatigue in $31.30 \%$ (36/115), and shortness of breath in $13.91 \%$ (16/115) of the patients (Supplementary Table 1). Thirdly, gastrointestinal symptoms were determined in the patients, and we found that $12.17 \%$
(14/115) of the cases had diarrhea, $7.83 \%$ (9/115) of the cases with nausea or vomiting, $7.83 \%(9 / 115)$ of the cases with inappetence (Supplementary Table 1). In addition, no significant differences in clinical manifestions were evident among different groups.

\section{Laboratory tests (peripheral blood routine examination and clinical biochemistry)}

Laboratory results of the patients containing peripheral blood routine examination and clinical biochemistry were summarized in Table 1 at the time of baseline evaluation. Firstly, blood routine examination of the patients was determined. Generally, the values of erythrocyte, leukocyte and platelet were within normal limit in most of COVID19 patients (Table 1). Lymphocytopenia was observed in $54.78 \%$ of the COVID-19 patients and lymphocytopenia occurred in $100 \%$ of critical cases. T cell subsets were also analyzed and the results showed that $\mathrm{CD} 3+, \mathrm{CD} 4+$ and $\mathrm{CD} 8+\mathrm{T}$ lymphocyte subsets were decreased in some of the COVID-19 patients. Compared with the ordinary group and the severe group, the proportion of $\mathrm{CD} 3+, \mathrm{CD} 4+$ and $\mathrm{CD} 8+\mathrm{T}$ cells decreased significantly in the critical group (Table 1). Secondly, the parameters of liver functional tests were analyzed. Most of the patients had normal levels of AST, ALT, ALP, $\gamma$ GT and total bilirubin. Significant difference in AST level was observed among different groups. Liver synthetic function was also assessed by the detection of albumin and PT, and we found that $60.00 \%$ (69/115) of the cases had hypoalbuminemia, $3.88 \%$ of the cases had increased prothrombin time. Thirdly, biomarkers of renal function indicated by serum urea and creatinine showed that most of the patients had normal renal function (Table 1). Fourthly, some biomarkers of metabolic syndrome were collected and the results showed that no significant differences in the levels of cholesterol (CHOL) and triglyceride (TG) were observed among different groups. Most of the patients had normal uric acid level; surprisingly, critical cases with the lowest level of uric acid (Table 1). Finally, abnormal CRP level was detected in $64.42 \%$ of the patients. Elevated AST level, hypoalbuminemia and increased CRP level were frequently found in critical cases.

\section{Imaging findings of chest and upper abdominal CT in COVID-19 patients}

Pneumonia in COVID-19 patients was commonly manifested as ground-glass opacity, smooth or irregular interlobular septal thickening, consolidation with air bronchogram, crazy-paving pattern, and pleura effusion (Figs. 2 and 3). Most of the patients suffered pneumonia in multiple lobes. The severity of pneumonia was evaluated by a semi-quantitative scoring system and the median score 
Table 1 Laboratory tests of patients with COVID-19 on admission

\begin{tabular}{|c|c|c|c|c|c|}
\hline & All patients & Ordinary & Severe & Critical & $p$ Value* \\
\hline \multicolumn{6}{|c|}{ Peripheral blood routine examination } \\
\hline Erythrocytes, $10^{12} / \mathrm{L}$ & $3.84(3.52-4.24)$ & $3.94(3.74-4.38)$ & $3.72(3.36-4.14)$ & $3.92(3.36-4.18)$ & 0.459 \\
\hline $\mathrm{RBC}<1 \times \mathrm{LLN}, \%(n / N)$ & $79.13 \%(91 / 115)$ & $74.00 \%(37 / 50)$ & $83.33 \%(40 / 48)$ & $82.35 \%(14 / 17)$ & \\
\hline Leukocyte, $10^{9} / \mathrm{L}$ & $5.20(4.06-6.77)$ & $5.21(3.92-6.38)$ & $4.66(3.87-6.63)$ & $7.23(5.04-9.56)$ & 0.138 \\
\hline $\mathrm{WBC}>1 \times \mathrm{ULN}, \%(n / N)$ & $5.22 \%(6 / 115)$ & 0 & $2.08 \%(1 / 48)$ & $29.41 \%(5 / 17)$ & \\
\hline $\mathrm{WBC}<1 \times \mathrm{LLN}, \%(n / N)$ & $13.04 \%(15 / 115)$ & $14.00 \%(7 / 50)$ & $14.58 \%(7 / 48)$ & $5.88 \%(1 / 17)$ & \\
\hline Platelet, $10^{9} / \mathrm{L}$ & $229.00(178.00-305.00)$ & $252.50(207.50-310.25)$ & $213.00(167.50-290.50)$ & $169.00(123.00-284.50)$ & 0.455 \\
\hline PLT $<1 \times \operatorname{LLN}, \%(n / N)$ & $7.83 \%(9 / 115)$ & $2.00 \%(1 / 50)$ & $8.33 \%(4 / 48)$ & $23.53 \%(4 / 17)$ & \\
\hline Lymphocytes, $10^{9} / \mathrm{L}$ & $1.04(0.80-1.41)$ & $1.23(0.90-1.65)$ & $1.04(0.81-1.41)$ & $0.61(0.45-0.87)$ & $<0.001$ \\
\hline $\mathrm{LY}<1 \times \mathrm{LLN}, \%(n / N)$ & $54.78 \%(63 / 115)$ & $38.00 \%(19 / 50)$ & $56.25 \%(27 / 50)$ & $100 \%(17 / 17)$ & \\
\hline $\mathrm{CD} 3+\mathrm{T}$ cells, $10^{9} / \mathrm{L}$ & $0.74(0.54-1.03)$ & $0.87(0.63-1.20)$ & $0.74(0.60-1.05)$ & $0.35(0.29-0.52)$ & $<0.001$ \\
\hline$<1 \times \operatorname{LLN}, \%(n / N)$ & $71.43 \%(80 / 112)$ & $90.47 \%(33 / 49)$ & $65.96 \%(31 / 47)$ & $100 \%(16 / 16)$ & \\
\hline CD4+ T cells, $10^{9} / \mathrm{L}$ & $0.32(0.22-0.48)$ & $0.35(0.26-0.53)$ & $0.34(0.24-0.49)$ & $0.14(0.10-0.22)$ & $<0.001$ \\
\hline$<1 \times \operatorname{LLN}, \%(n / N)$ & $81.25 \%(91 / 112)$ & $77.55 \%(38 / 49)$ & $78.72 \%(37 / 47)$ & $100 \%(16 / 16)$ & \\
\hline $\mathrm{CD} 8+\mathrm{T}$ cells, $10^{9} / \mathrm{L}$ & $0.17(0.10-0.25)$ & $0.18(0.12-0.28)$ & $0.18(0.13-0.28)$ & $0.07(0.04-0.09)$ & $<0.001$ \\
\hline$<1 \times \operatorname{LLN}, \%(n / N)$ & $81.25 \%(91 / 112)$ & $79.59 \%(39 / 49)$ & $76.60 \%(36 / 47)$ & $100 \%(16 / 16)$ & \\
\hline \multicolumn{6}{|l|}{ Biochemistry } \\
\hline ALT, U/L & $27.00(19.00-45.00)$ & $27.00(16.75-50.50)$ & $27.00(20.00-41.00)$ & $26.00(18.00-55.00)$ & 0.938 \\
\hline ALT $>1 \times \mathrm{ULN}, \%(n / N)$ & $27.83 \%(32 / 115)$ & $30.00 \%(15 / 50)$ & $25.00 \%(12 / 48)$ & $29.41 \%(5 / 17)$ & \\
\hline AST, U/L & $28.00(20.00-40.00)$ & $23.50(16.00-34.50)$ & $30.00(21.25-40.75)$ & $37.00(27.00-46.00)$ & 0.014 \\
\hline AST $>1 \times \mathrm{ULN}, \%(n / N)$ & $22.61 \%(26 / 115)$ & $14.00 \%(7 / 50)$ & $25.00 \%(12 / 48)$ & $41.18 \%(7 / 17)$ & \\
\hline ALP, U/L & $65.00(52.00-80.00)$ & $65.50(50.75-84.25)$ & $63.00(54.25-77.75)$ & $65.00(53.00-91.00)$ & 0.796 \\
\hline ALP $>1 \times \operatorname{ULN}, \%(n / N)$ & $5.22 \%(6 / 115)$ & $4.00 \%(2 / 50)$ & $4.17 \%(2 / 48)$ & $11.76 \%(2 / 17)$ & \\
\hline$\gamma \mathrm{GT}, \mathrm{U} / \mathrm{L}$ & $27.00(17.00-49.00)$ & $26.00(15.00-49.00)$ & $26.50(18.00-36.50)$ & $42.00(21.50-100)$ & 0.188 \\
\hline$\gamma \mathrm{GT}>1 \times \mathrm{ULN}, \%(n / N)$ & $27.83 \%(32 / 115)$ & $32.00 \%(16 / 50)$ & $16.67 \%(8 / 48)$ & $47.06 \%(8 / 17)$ & \\
\hline $\mathrm{T}$-BIL, $\mu \mathrm{mol} / \mathrm{L}$ & $11.20(8.70-14.40)$ & $10.80(7.78-14.40)$ & $12.15(9.83-14.38)$ & $11.10(8.95-22.50)$ & 0.288 \\
\hline $\mathrm{T}-\mathrm{BIL}>1 \times \mathrm{UL} N, \%(n / N)$ & $13.04 \%(15 / 115)$ & $12.00 \%(6 / 50)$ & $8.33 \%(4 / 48)$ & $29.41 \%(5 / 17)$ & \\
\hline ALB, g/L & $34.10(31.60-37.30)$ & $35.20(32.88-38.30)$ & $33.50(30.83-37.03)$ & $32.20(28.15-34.65)$ & 0.003 \\
\hline ALB $<1 \times \operatorname{LLN}, \%(n / N)$ & $60.00 \%(69 / 115)$ & $46.00 \%(23 / 50)$ & $64.58 \%(31 / 48)$ & $88.24 \%(15 / 17)$ & \\
\hline PT, S & $13.40(12.90-14.20)$ & $13.30(12.90-13.85)$ & $13.65(13.03-14.20)$ & $13.70(12.95-15.35)$ & 0.290 \\
\hline $\mathrm{PT}>1 \times \mathrm{ULN}, \%(n / N)$ & $3.88 \%(4 / 103)$ & $0(0 / 41)$ & $2.17 \%(1 / 46)$ & $18.75 \%(3 / 16)$ & \\
\hline INR & $1.04(0.99-1.12)$ & $1.03(0.99-1.09)$ & $1.07(1.00-1.12)$ & $1.07(1.00-1.24)$ & 0.294 \\
\hline $\mathrm{INR}>1 \times \mathrm{ULN}, \%(n / N)$ & $3.88 \%(4 / 103)$ & $0(0 / 41)$ & $2.17 \%(1 / 46)$ & $18.75 \%(3 / 16)$ & \\
\hline Urea, $\mu \mathrm{mol} / \mathrm{L}$ & $3.92(3.10-5.05)$ & $3.66(2.76-4.53)$ & $4.00(3.23-5.32)$ & $3.95(3.45-5.87)$ & 0.138 \\
\hline Urea $>1 \times$ ULN, $\%(n / N)$ & $26.09 \%(30 / 115)$ & $28.00 \%(14 / 50)$ & $25.00 \%(12 / 48)$ & $23.53 \%(4 / 17)$ & \\
\hline $\mathrm{Cr}, \mu \mathrm{mol} / \mathrm{L}$ & $68.10(56.90-77.80)$ & $62.35(54.73-73.13)$ & $71.40(63.50-83.98)$ & $63.90(54.30-80.50)$ & 0.011 \\
\hline $\mathrm{Cr}>1 \times \mathrm{ULN}, \%(n / N)$ & $5.22 \%(6 / 115)$ & $4.00 \%(2 / 50)$ & $6.25 \%(3 / 48)$ & $5.88 \%(1 / 17)$ & \\
\hline Uric acid, $\mu \mathrm{mol} / \mathrm{L}$ & $228.00(184.20-295.60)$ & $238.20(188.25-295.15)$ & $235.60(189.83-329.98)$ & $211.70(133.35-233.90)$ & 0.017 \\
\hline $\mathrm{UA}>1 \times \mathrm{ULN}, \%(n / N)$ & $3.48 \%(4 / 115)$ & 0 & $8.33 \%(4 / 48)$ & 0 & \\
\hline $\mathrm{CRP}, \mathrm{mg} / \mathrm{L}$ & $22.10(3.28-67.15)$ & $10.20(3.14-29.5)$ & $15.30(3.39-69.50)$ & $81.00(62.20-156.00)$ & $<0.001$ \\
\hline $\mathrm{CRP}>1 \times \mathrm{ULN}, \%(n / N)$ & $64.42 \%(67 / 104)$ & $55.32 \%(26 / 47)$ & $60.00 \%(24 / 40)$ & $100 \%(17 / 17)$ & \\
\hline $\mathrm{CHOL}, \mathrm{mmol} / \mathrm{L}$ & $3.92(3.42-4.39)$ & $3.93(3.38-4.81)$ & $3.96(3.44-4.32)$ & $3.76(3.51-4.73)$ & 0.993 \\
\hline $\mathrm{CHOL}>1 \times \mathrm{UL} N, \%(n / N)$ & $15.38 \%(16 / 104)$ & $20.00 \%(9 / 45)$ & $11.36 \%(5 / 44)$ & $13.33 \%(2 / 15)$ & \\
\hline $\mathrm{TG}, \mathrm{mmol} / \mathrm{L}$ & $1.18(0.91-1.53)$ & $1.24(0.96-1.60)$ & $1.15(0.84-1.56)$ & $1.27(0.85-1.51)$ & 0.618 \\
\hline $\mathrm{TG}>1 \times \mathrm{ULN}, \%(n / N)$ & $14.56 \%(15 / 103)$ & $20.00 \%(9 / 45)$ & $11.36 \%(5 / 44)$ & $7.14 \%(1 / 14)$ & \\
\hline
\end{tabular}

Continuous variables are presented as median (25th-75th percentiles), and categorical variables are presented as count (percentage)

*The $p$ values refer to Kruskal-Wallis $H$ test among different groups. Normal ranges: erythrocytes $(\mathrm{RBC})$ : $4.3-5.8 \times 10^{12} / \mathrm{L}$; leukocyte (WBC): $3.5-9.5 \times 10^{9} / \mathrm{L}$; platelet (PLT): $125-350 \times 10^{9} / \mathrm{L}$; lymphocytes $(\mathrm{LY}): 1.1-3.2 \times 10^{9} / \mathrm{L} ; \mathrm{CD} 3+\mathrm{T}$ cells: $\geq 0.995 \times 10^{9} / \mathrm{L} ; \mathrm{CD} 4+\mathrm{T}$ cells: $\geq 0.550 \times 10^{9} / \mathrm{L} ; \mathrm{CD} 8+\mathrm{T}$ cells: $\geq 0.320 \times 10^{9} / \mathrm{L}$; alanine aminotransferase (ALT): $5-40 \mathrm{U} / \mathrm{L}$; aspartate aminotransferase (AST): $8-40 \mathrm{U} /$ $\mathrm{L}$; alkaline phosphatase (ALP):40-150 U/L; $\gamma$-glutamyl transpeptidase $(\gamma$-GT): 11-50U/L; total bilirubin (T-BIL): $5.1-19.0 \mu \mathrm{mol} / \mathrm{L}$; albumin (ALB): $35-55 \mathrm{~g} / \mathrm{L}$; prothrombin time (PT): 11.0-16.0S; international normalized ratio(INR): $0.80-1.31$; urea: $2.9-8.2 \mathrm{mmol} / \mathrm{L}$; creatinine(Cr): 44.0-106.0 $\mu \mathrm{mol} / \mathrm{L}$; C-reactive protein $(\mathrm{CRP}):<8 \mathrm{mg} / \mathrm{L}$; uric acid (UA): $208-428 \mu \mathrm{mol} / \mathrm{L}$; cholesterol $(\mathrm{CHOL}):<5.2 \mathrm{mmol} / \mathrm{L}$; triglycerides (TG): $<1.7 \mathrm{mmol} / \mathrm{L}$ 

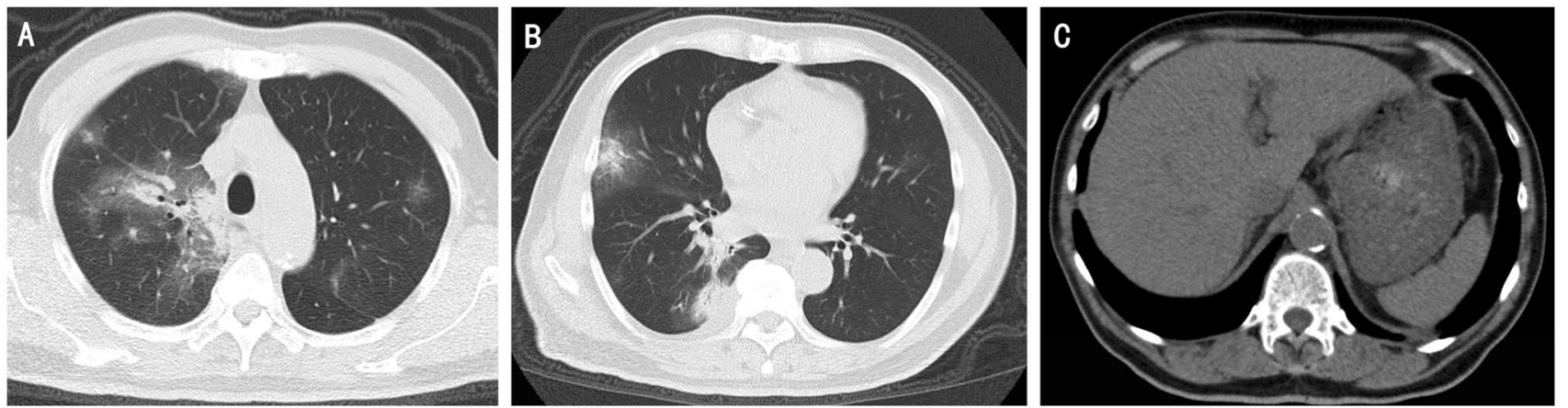

Fig. 2 CT findings in an 80-year-old male patient with severe COVID-19. a and b Transverse thin-section chest CT scan showed ground-glass opacities in both sides, partial consolidation and small amount of pleural effusion in right side, semi-quantitative score of pulmonary lesions was 10: $\mathbf{c}$ transverse thin-section CT scan revealed homogeneous liver hypodensity, the L/S attenuation ratio is 0.99
Fig. 3 CT findings in a 51-yearold male patient with critical COVID-19 who had type I respiratory failure and received mechanical ventilation. a and b transverse thin-section chest CT scan showed ground-glass opacities, consolidation with air bronchogram and pleural effusion in both sides, semi-quantitative score of lung lesions was 22; c transverse thin-section CT scan showed heterogeneous liver hypodensity, the L/S attenuation ratio was 1.04 ; $\mathbf{d}$ enlarged view of the gallbladder showed pericholecystic fat stranding (arrows)
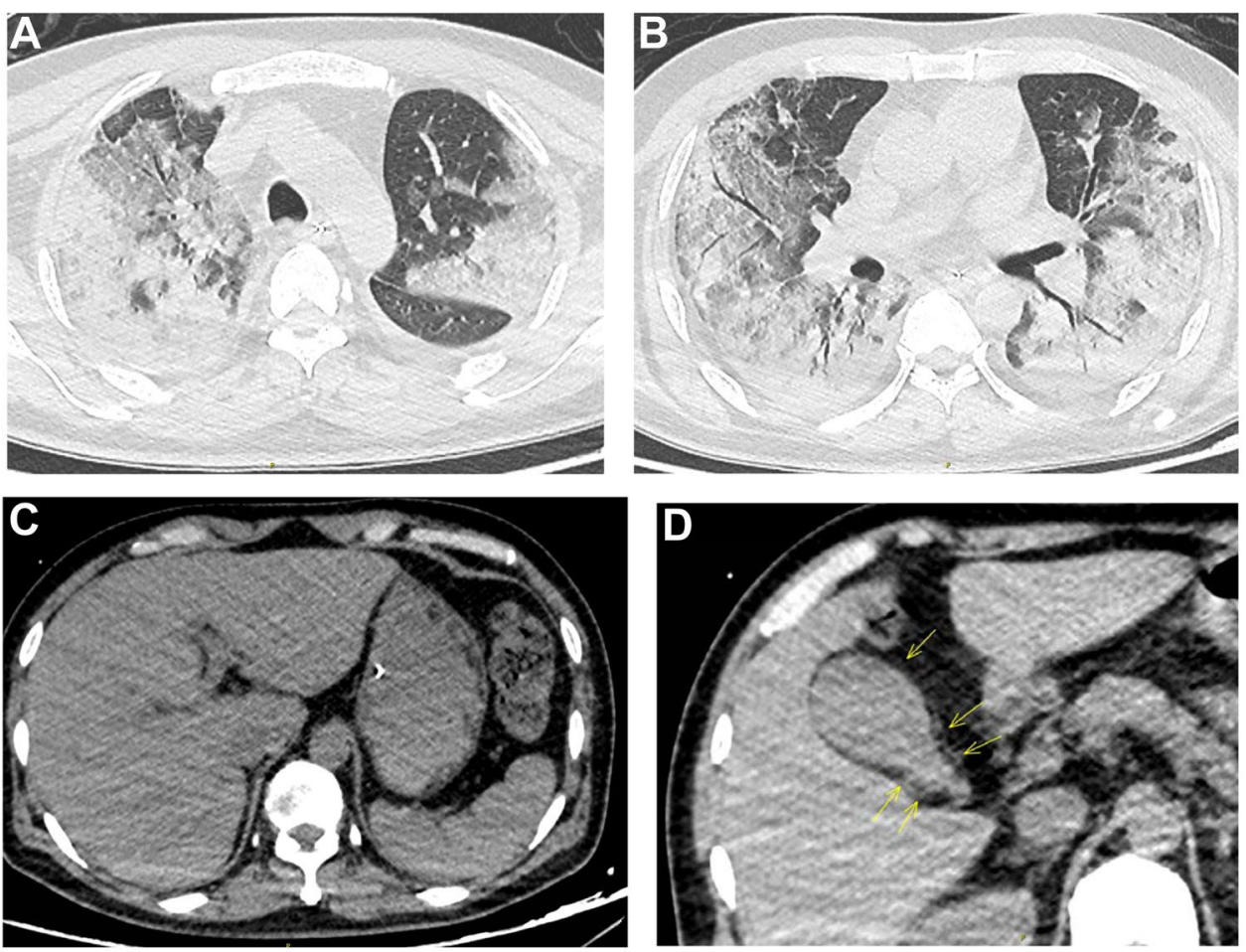

of lung involvement was 11 (IQR: 8-15) (Table2). More importantly, significant difference in semi-quantitative scores of lung involvement was observed among different groups (Table2). Then, imaging features of abdominal CT were determined. Common CT findings in upper abdominal CT scan were liver hypodensity and pericholecystic fat stranding (Table 2). Hepatomegaly, splenomegaly, periportal edema, edema of the gallbladder walls, periportal lymphadenopathy, biliary dilatation and ascites were not observed. Homogeneous or heterogeneous hepatic hypodensity was the most common CT finding (Figs. 2 and 3). Liver hypodensity was observed in $26.09 \%$ (30/115) of the patients and statistical analysis revealed significant difference among different groups (Table2). Moreover, the liver-to-spleen $(\mathrm{L} / \mathrm{S})$ attenuation ratio was measured and the interobserver agreement for assessment of $\mathrm{L} / \mathrm{S}$ attenuation ratios was very good $(\mathrm{ICC}=0.98)$. A decrease in the $\mathrm{L} / \mathrm{S}$ attenuation ratio $(<1)$ was detected in $16.52 \%$ of the whole cohort, up to $47.06 \%$ of critical cases. Meanwhile, significant difference in the L/S attenuation ratio was observed among different groups (Table2). Finally, pericholecystic fat stranding was evaluated. Unfortunately, 21 cases were excluded due to the patients with cholecystolithiasis or cholecystectomy. Pericholecystic fat stranding was observed in $21.27 \%$ (21/94) of the patients (Fig. 3), but no significant difference was evident among different groups. 
Table 2 Baseline radiologic findings of patients with COVID-19

\begin{tabular}{|c|c|c|c|c|c|}
\hline & All Patients & Ordinary & Severe & Critical & $p$ Value \\
\hline \multicolumn{6}{|l|}{ Radiologic features of upper abdominal CT } \\
\hline Liver hypodensity, \% $(n / N)$ & $26.09 \%(30 / 115)$ & $14.00 \%(7 / 50)$ & $27.08 \%(13 / 48)$ & $58.8 \%(10 / 17)$ & $0.002^{\#}$ \\
\hline Pericholecystic fat stranding & $21.27 \%(20 / 94)$ & $20.00 \%(8 / 40)$ & $22.50 \%(9 / 40)$ & $21.43 \%(3 / 14)$ & $1^{\#}$ \\
\hline \multicolumn{6}{|l|}{ Quantitative analysis of liver hypodensity } \\
\hline $\mathrm{L} / \mathrm{S}$ attenuation ratio & $1.21(1.06-1.30)$ & $1.24(1.15-1.34)$ & $1.21(1.06-1.32)$ & $1.01(0.90-1.11)$ & $<0.001 *$ \\
\hline $\mathrm{L} / \mathrm{S}$ attenuation ratio, $\%(n / N)^{\mathrm{a}}$ & $16.52 \%(19 / 115)$ & $6.00 \%(3 / 50)$ & $16.67 \%(8 / 48)$ & $47.06 \%(8 / 17)$ & $0.001^{\#}$ \\
\hline \multicolumn{6}{|l|}{ Radiologic features of chest CT } \\
\hline CT scores of pulmonary involvements & $11(8-15)$ & $9.5(7-13)$ & $11(9-15)$ & $20(14-24)$ & $<0.001^{*}$ \\
\hline Pleural effusion & $27.83 \%(32 / 115)$ & $12.00 \%(6 / 50)$ & $39.58(19 / 48)$ & $41.18 \%(7 / 17)$ & $0.003^{\#}$ \\
\hline
\end{tabular}

Continuous variables are presented as median (25th-75th percentiles), and categorical variables are presented as count (percentage)

$L / S$ liver-to-spleen

*The $p$ values refer to Kruskal-Wallis $H$ test among different groups

\#The $p$ values refer to Fisher exact test among different groups

${ }^{\mathrm{a}} \mathrm{L} / \mathrm{S}$ attenuation ratio $<1$

\section{Correlation of severity grading with clinical manifestations, radiologic features and liver function tests}

To evaluate the value of radiological features and liver function tests, the correlations of severity grading with clinical manifestations, CT features and liver function tests were determined. Firstly, the correlations of clinical manifestation with a panel of liver function tests and severity grading were investigated in patients with COVID-19 and the results showed no correlation of clinical manifestations with pulmonary lesions, severity grading and liver functional tests (Table S2); secondly, the correlations of semi-quantitative scoring of pulmonary lesions with a panel of liver function tests and severity grading were investigated in patients with COVID-19, and the correlation tests demonstrated that the score of pulmonary lesions was associated with liver function tests, severity grading and CRP (Table3). Thirdly, the correlations of severity grading with a panel of liver function tests, CRP and pulmonary lesions were determined. The results demonstrated the severity correlated with AST, albumin, CRP, semi-quantitative CT-scoring of pulmonary lesions and CT-quantified liver/spleen attenuation ratio (Table3).

\section{Discussion}

COVID-19 has turned into a global pandemic disease. Previous studies demonstrated coronavirus COVID-19 (SARSCoV-2) resulted in liver injury in the whole cohort [8-10], we described clinical profiles and abdominal radiological features of COVID-19 patients according to severity grading, and then determined the correlation of radiological features with clinical profiles. Abnormal liver function tests were observed in some of COVID-19 patients, radiological signs of liver in non-contrast $\mathrm{CT}$ scan included liver hypodensity and pericholecystic fat stranding. The correlation tests demonstrated that disease severity correlated with AST, albumin, CRP, the score of pulmonary lesions, the L/S attenuation ratio. Thus, it will allow an earlier identification of high-risk patients for immediate effective intervention in future.

Gastrointestinal symptoms of patients with COVID-19 were analyzed, and common GI symptoms included diarrhea, nausea, vomiting, and inappetence. The proportion of COVID-19 patients with GI symptoms in our study was similar to that of previous research [7]. GI symptoms in patients with COVID-19 may result from the infection of COVID-19 in intestinal tissues [7]. In addition, the interaction between brain and gut might cause discomfort to the gastrointestinal tract. The results of peripheral routine blood examination showed the values of erythrocyte, leukocyte and platelet were within normal limit in most of the patients. Some of the patients had lymphocytopenia, especially CD3+ T cells. $\mathrm{CD} 3+\mathrm{T}$ cells are mainly composed of CD4+ $\mathrm{T}$ and $\mathrm{CD} 8+\mathrm{T}$ cells, CD4+ T and CD8+ $\mathrm{T}$ cells are critical for mediating clearance following many acute viral infections in the lung, which leads to a decrease in CD4+ T and CD8+ T cells [23, 24]. A panel of liver functional tests was determined, and the result showed that some of the patients had abnormal liver function, and clinically significant liver injury was uncommon even in critical cases. The mechanism underlying liver damage has not been explored until now. Liver damage in the COVID-19 patients might be caused by viral infection of liver cells, adverse drug reactions, systemic inflammation, underlying liver diseases, hypoxic hepatitis, etc. [3-5]. Xu et al. reported the drugs used for COVID-19 


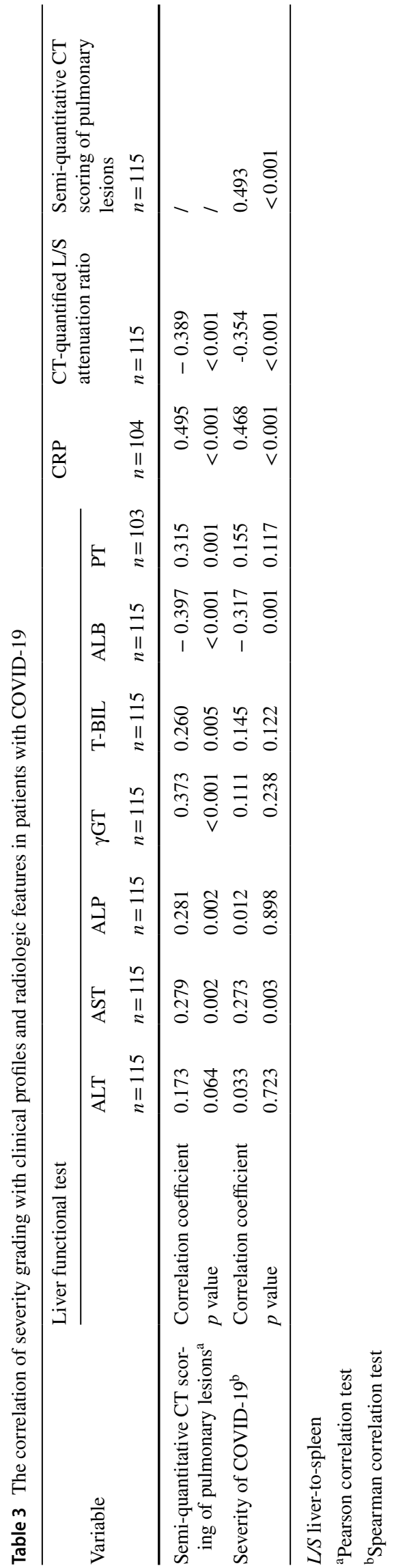

patients probably induced liver damage, such as antibiotics, antiviral agents, etc.[5]. Thus, to exclude drug-induced liver injury (DILI), liver functional tests were collected within 2 days after admission. In addition, liver dysfunction occurred in patients with chronic liver diseases, so the patients with chronic liver diseases were excluded in our study. Thus, we speculate liver injury in COVID-19 patients might result from viral infection of liver cells, inflammatory responses, and hypoxic hepatitis in our study. Preliminary studies showed that angiotensin-converting enzyme 2 (ACE2) receptor expression is enriched in cholangiocytes [25] and SARS-CoV-2 enters the target cell through ACE2 receptor [25], which results in abnormality of serum $\gamma \mathrm{GT}$, a diagnostic biomarker for cholangiocyte injury. Abnormal PT and hypoalbuminemia were found in some of COVID19 patients. Severe COVID-19 frequently induces hypercoagulability with both microangiopathy and local thrombus formation; abnormality in prothrombin time is relatively uncommon in initial presentations [26, 27]. Abnormal PT might result from liver injury or coagulopathy. Hypoalbuminemia is caused by liver dysfunction, heart failure, kidney damage, protein-losing enteropathy, and malnutrition. In our study, heart failure, kidney damage, protein-losing enteropathy were not observed in our study. Thus, hypoalbuminemia would be caused by malnutrition or liver dysfunction. Interestingly, hypoalbuminemia and increased AST level were frequently found in critical cases, and correlation analysis revealed the correlations of albumin and AST level with severity grading. Thus, biomarkers of liver function containing AST and albumin should be detected in patients with COVID-19.

Common findings of upper abdominal CT scan in the patients with COVID-19 were mainly manifested as homogeneous or heterogeneous liver hypodensity and pericholecystic fat stranding. Further study revealed the L/S attenuation ratio and pulmonary lesions correlated positively with the severity of the disease. These indicated that the infection of SARS-CoV-2 may result in multiple organ dysfunction syndrome (MODS) containing liver injury. Pathological examination of liver showed moderate steatosis and mild lobular and portal activity in a patient with COVID-19 [12]. Liver hypodensity was a common sign of liver steatosis or acute hepatitis, while other imaging signs of acute hepatitis, such as hepatomegaly, periportal edema, edema of the gallbladder wall, and periportal lymphadenopathy were not observed in patients with COVID-19. Thus, liver hypodensity and a decrease in the L/S attenuation ratio might be attributed to liver steatosis. Obviously, transient elastography (FibroScan) with controlled attenuation parameter (CAP) has demonstrated good accuracy in quantifying the levels of liver steatosis; however, most of the patients did not receive Fibroscan due to mild liver injury and uncovered medical procedures. The etiologies of hepatic steatosis 
include NAFLD, alcoholism, hepatitis C infection, some drugs, autoimmune disease, malnutrition, etc. Liver steatosis resulting from NAFLD should be excluded. Some of clinical profiles in the COVID-19 patients were different from those of NAFLD patients. Firstly, the enrolled patients had normal value for ALT and AST before hospital admission. Thus, liver damage was not caused by chronic liver diseases, such as NAFLD. Secondly, elevated levels of liver enzymes are often seen in NAFLD, hypoalbuminemia is often observed in COVD-19 patients. Thirdly, liver hypodensity on CT scans was improved within a short time in some COVID-19 patients (Fig. S2); however, the improvement of CT sign needs a long period of time in NAFLD. Fourthly, no significant differences in plasma lipid levels were observed among different groups of the COVID-19 patients divided by the severity. Thus, the hypothesis is supported by indirect evidences and it should be confirmed by a large sample of pathological examinations in future. More importantly, the mechanism underlying liver steatosis should be explored in the patients infected with SARS-CoV-2. In addition, pericholecystic fat stranding, a common sign of abdominal CT scan, might be associated with inflammation. Interestingly, enlargement of hilar lymph nodes and spleen were not found.

The correlations of clinical profiles and radiological signs with severity grading were investigated, and the results showed severity grading correlated with AST, albumin, CRP, semi-quantitative scoring of pulmonary lesions, the liver-to-spleen (L/S) attenuation ratio. These indicated elevated AST level, hypoalbuminemia, a high CRP, a low L/S attenuation ratio, a high score of pulmonary lesions might be indicators of severe or critical cases. Our study demonstrated the severity of SARS-CoV-2 infection correlated with $\mathrm{CRP}$ and pulmonary lesions, which was similar to previous studies. Interestingly, the severity correlates with AST, CT-quantified liver/spleen attenuation ratio in patients with COVID-19. Elevated AST can be caused by liver injury, cardiovascular disease, kidney diseases, muscle disorders, etc. Elevated AST might result from multiple organ dysfunction, which is associated with the severity of SARS-CoV-2 infection. CT-quantified liver/spleen attenuation ratio revealed the severity of liver damage, which was related to multiple organ dysfunction syndrome.

Obviously, our study had several limitations. Firstly, it was a single-center retrospective study. Selection bias occurred in retrospective cohort studies, the patients with mild system and normal lung manifestation were excluded in our study. In addition, most of the enrolled patients were middle-aged and aged people. Secondly, the patients did not receive contrast-enhanced CT scan. Thirdly, limited number of the cases in our cohort might have resulted in the bias of the results. Finally, follow-up data of the patients were not provided. At the end of the 2-month follow-up, 106 patients survived, nine patients died. We analyzed the prognosis of
COVID-19 patients; unfortunately, we did not get useful data due to small sample size and relatively few deaths. In conclusion: COVID-19 patients display abdominal and pulmonary CT imaging abnormalities and some of the patients have abnormal liver function. Furthermore, some of CT signs and CRP are positively correlated with severity grading; thus, it will allow an earlier identification of high-risk patients. Further studies should be performed to confirm our conclusion and develop suitable therapeutic strategies, and investigate the prognosis in future.

Acknowledgements We thank Dr Hui Guo (Department of Breast and Thyroid Surgery, Union Hospital, Tongji Medical College, HUST), Dr Cen Chen (Department of Radiology, Union Hospital, Tongji Medical College, HUST) for kind assistance in the collection of laboratory data, and Mr. Bo Rao (School of Electric and Electronical Engineering, HUST), Dr Yu Shi (Departments of Radiology, Shengjing Hospital, China Medical University) for helpful suggestions in statistical analysis.

Author contributions XL and YS designed the study; PL, LZ, XL, QT collected data; PL, LZ, XL, FY reviewed CT imaging; XL, YS, PH, $\mathrm{CZ}, \mathrm{YH}$ interpreted the data; LZ, HS performed statistical analysis; PL, LZ, XL, YS analyzed the data, reviewed the chart, interpreted the data, and wrote the paper.

Funding This work was supported by the Key Special Project of Ministry of Science and Technology, China (No. 2020YFC0845700), the Emergency Projects of Science and Technology Department of Hubei Province (No.2020FCA014); COVID-19 Rapid Response Call of Huazhong University of Science and Technology (No.2020kfyXGYJ021 and 2020kfyXGYJ026), and National Natural Science Foundation of China (81570555).

\section{Compliance with ethical standards}

Conflict of interest Ping Lei, Lan Zhang, Ping Han, Chuansheng Zheng, Qiaoxia Tong, Haitao Shang, Fan Yang, Yu Hu, Xin Li, Yuhu Song disclose no conflicts.

Ethical approval This study was conducted in accordance with the Declaration of Helsinki. Our study was approved by Ethics Committee of Union Hospital, Tongji Medical College, Huazhong University of Science and Technology (No2020-0312).

Informed consent Informed consent was waived because of the retrospective nature of the study and the usage of anonymous clinical data.

\section{References}

1. Chen N, Zhou M, Dong X, et al. Epidemiological and clinical characteristics of 99 cases of 2019 novel coronavirus pneumonia in Wuhan, China: a descriptive study. Lancet 2020;395:507-513.

2. Rismanbaf A, Zarei S. Liver and kidney injuries in COVID-19 and their effects on drug therapy; a letter to editor. Arch Acad Emerg Med 2020;8:e17.

3. Bangash MN, Patel J, Parekh D. COVID-19 and the liver: little cause for concern. Lancet Gastroenterol Hepatol 2020. https://doi. org/10.1016/S2468-1253(20)30084-4. 
4. Zhang C, Shi L, Wang FS. Liver injury in COVID-19: management and challenges. Lancet Gastroenterol Hepatol 2020. https:// doi.org/10.1016/S2468-1253(20)30057-1.

5. Xu L, Liu J, Lu M, et al. Liver injury during highly pathogenic human coronavirus infections. Liver Int 2020. https://doi. org/10.1111/liv.14435.

6. Wang T, Du Z, Zhu F, et al. Comorbidities and multi-organ injuries in the treatment of COVID-19. Lancet 2020. https://doi. org/10.1016/S0140-6736(20)30558-4.

7. Zhou Z, Zhao N, Shu Y, et al. Effect of gastrointestinal symptoms on patients infected with COVID-19. Gastroenterology 2020. https ://doi.org/10.1053/j.gastro.2020.03.020.

8. Huang C, Wang Y, Li X, et al. Clinical features of patients infected with 2019 novel coronavirus in Wuhan, China. Lancet 2020;395:497-506.

9. Guan WJ, Ni ZY, Hu Y, et al. Clinical characteristics of Coronavirus disease 2019 in China. N Engl J Med 2020;382:1708-1722.

10. Wang D, Hu B, Hu C, et al. Clinical characteristics of 138 hospitalized patients with 2019 novel Coronavirus-infected pneumonia in Wuhan, China. JAMA 2020. https://doi.org/10.1001/ jama.2020.1585.

11. Fan Z, Chen L, Li J, et al. Clinical features of COVID19-related liver damage. medRxiv 2020. https://doi. org/10.1101/2020.02.26.20026971

12. Xu Z, Shi L, Wang Y, et al. Pathological findings of COVID-19 associated with acute respiratory distress syndrome. Lancet Respir Med 2020. https://doi.org/10.1016/S2213-2600(20)30076-X.

13. National Health Commission of the People's Republic of China and National Administration of Traditional Chinese Medicine of the People's Republic of China. Diagnosis and treatment plan of Corona Virus disease 2019 (tentative sixth edition). Global Health J. https://doi.org/10.1016/j.glohj.2020.03.001

14. Fang Y, Zhang H, Xie J, et al. Sensitivity of Chest CT for COVID-19: Comparison to RT-PCR. Radiology 2020. https:// doi.org/10.1148/radiol.2020200432.

15. Shi H, Han X, Jiang N, et al. Radiological findings from 81 patients with COVID-19 pneumonia in Wuhan, China: a descriptive study. Lancet Infect Dis 2020. https://doi.org/10.1016/S1473 -3099(20)30086-4.

16. Wang Y, Dong $\mathrm{C}, \mathrm{Hu} \mathrm{Y}$, et al. Temporal changes of $\mathrm{CT}$ findings in 90 patients with COVID-19 pneumonia: a longitudinal study. Radiology 2020. https://doi.org/10.1148/radiol.2020200843.

17. Kan X, Ye J, Rong X, et al. Diagnostic performance of contrastenhanced CT in pyrrolizidine alkaloids-induced hepatic sinusoidal obstructive syndrome. Sci Rep 2016;6:37998.
18. Li X, Yang X, Xu D, et al. Magnetic resonance imaging findings in patients with pyrrolizidine alkaloid-induced hepatic sinusoidal obstruction syndrome. Clin Gastroenterol Hepatol 2017;15:955-957.

19. Guo T, Li X, Yang X, et al. Gadoxetic acid-enhanced hepatobiliary-phase magnetic resonance imaging for pyrrolizidine alkaloidinduced hepatic sinusoidal obstruction syndrome and association with liver function. Sci Rep 2019;9:1231.

20. Chang Y-C, Yu C-J, Chang S-C, et al. Pulmonary sequelae in convalescent patients after severe acute respiratory syndrome evaluation with thin section CT. Radiology 2005;236:1067-1075.

21. Pan F, Ye T, Sun P, et al. Time course of lung changes on chest CT during recovery from 2019 novel Coronavirus (COVID-19) pneumonia. Radiology 2020.https://doi.org/10.1148/radiol.20202 00370

22. Zeb I, Li D, Nasir K, et al. Computed tomography scans in the evaluation of fatty liver disease in a population based study: the multi-ethnic study of atherosclerosis. Acad Radiol 2012;19:811-818.

23. Chen G, Wu D, Guo W, et al. Clinical and immunological features of severe and moderate coronavirus disease 2019. J Clin Invest 2020;130:2620-2629.

24. Qin C, Zhou L, Hu Z, et al. Dysregulation of immune response in patients with COVID-19 in Wuhan. China. Clin Infect Dis 2020. https://doi.org/10.1093/cid/ciaa248.

25. Hofmann M, Kleine-Weber H, Krüger N, et al. The novel coronavirus 2019 (2019-nCoV) uses the SARS-coronavirus receptor ACE2 and the cellular protease TMPRSS2 for entry into target cells. bioRxiv 2020. https://doi.org/10.1101/2020.01.31.929042

26. Connors JM, Levy JH. COVID-19 and its implications for thrombosis and anticoagulation. Blood 2020;135:2033-2040.

27. Iba T, Levy JH, Levi M, et al. Coagulopathy of Coronavirus disease 2019. Crit Care Med 2020. https://doi.org/10.1097/ CCM.0000000000004458.

Publisher's Note Springer Nature remains neutral with regard to jurisdictional claims in published maps and institutional affiliations. 